\title{
Exosomes derived from human menstrual blood-derived stem cells alleviate fulminant hepatic failure
}

\author{
Lu Chen, Bingyu Xiang, Xiaojun Wang and Charlie Xiang ${ }^{*}$
}

\begin{abstract}
Background: Human menstrual blood-derived stem cells (MenSCs) are a novel source of MSCs that provide the advantage of being easy to collect and isolate. Exosomes contain some mRNAs and adhesion molecules that can potentially impact cellular and animal physiology. This study aimed to investigate the therapeutic potential of MenSC-derived exosomes (MenSC-Ex) on AML12 cells (in vitro) and D-GaIN/LPS-induced FHF mice (in vivo).

Methods: Transmission electron microscopy and Western blot were used to identify MenSC-Ex. Antibody array was used to examine cytokine levels on MenSC-Ex. MenSC-Ex were treated in D-GalN/LPS-induced AML12 in vitro. Cell proliferation and apoptosis were measured. MenSC-Ex were injected into the tail veins of mice $24 \mathrm{~h}$ before treatment with D-GalN/LPS. Blood and liver tissues served as physiological and biochemical indexes. The number of liver mononuclear cells (MNCs) and the amount of the active apoptotic protein caspase-3 were determined to elaborate the mechanism of hepatoprotective activity.
\end{abstract}

Results: Human menstrual blood-derived stem cell-derived exosomes (MenSC-Ex) are bi-lipid membrane vesicles that have a round, ball-like shape with a diameter of approximately 30-100 $\mathrm{nm}$. Cytokine arrays have shown that MenSC-Ex expressed cytokines, including ICAM-1, angiopoietin-2, Axl, angiogenin, IGFBP-6, osteoprotegerin, IL-6, and IL-8. MenSC-Ex markedly improved liver function, enhanced survival rates, and inhibited liver cell apoptosis at $6 \mathrm{~h}$ after transplantation. MenSC-Ex migrated to sites of injury and to AML12 cells (a mouse hepatocyte cell line), respectively. Moreover, MenSC-Ex reduced the number of liver mononuclear cells (MNCs) and the amount of the active apoptotic protein caspase-3 in injured livers.

Conclusions: In conclusion, our results provide preliminary evidence for the anti-apoptotic capacity of MenSC-Ex in FHF and suggest that MenSC-Ex may be an alternative therapeutic approach to treat FHF.

Keywords: D-GalN/LPS, Exosome, Menstrual blood-derived stem cell

\section{Background}

Clinical fulminant hepatic failure (FHF) causes relatively high mortality and affected patients present with very severe clinical symptoms, such as coagulopathy, jaundice, and multiorgan failure. However, the only clinical treatment for FHF is liver transplantation, which is limited by a shortage of donor livers [1].

D-galactosamine (D-GalN) and lipopolysaccharide (LPS) co-induce (D-GalN/LPS) FHF in mice, producing a

\footnotetext{
* Correspondence: cxiang@zju.edu.cn

State Key Laboratory for Diagnosis and Treatment of Infectious Diseases, and Collaborative Innovation Center for Diagnosis and Treatment of Infectious Diseases, School of Medicine, Zhejiang University, Hangzhou 310003, China
}

phenotype that copies clinical FHF. This mouse model is therefore commonly used as a test model [2]. LPS is the main component of Gram-negative bacterial cell walls, which induce very strong immunogenicity and can enhance immune response. When D-GalN/LPS are applied in mice, LPS activates immune cells in the liver, including monocytes, macrophages, and hepatic Kupffer cells [3].

Human menstrual blood-derived stem cells (MenSCs) are human menstrual blood progenitor cells (MBPCs) that are isolated from menstrual fluids [4-6]. MenSCs are similar to mesenchymal stem cells (MSCs), which have proliferative capabilities and broad multipotency, including the ability to differentiate into cell types belonging to 
all three germ lineages [6-8]. MenSCs exhibit higher proliferation rates than bone marrow-derived MSCs and can be easily obtained without invasive procedures [9-11]. In particular, our and other groups have reported that MenSCs induce low-level immunogenicity in clinical studies and can be expanded through at least 20 passages without genetic abnormalities $[9,12,13]$. The therapeutic potential of MenSCs has been demonstrated in several disease models, such as Duchenne muscular dystrophy, stroke, type 1 diabetes, premature ovarian failure, and myocardial infarction. These studies have suggested that MenSC-based therapies may be developed into future clinical applications [12, 14-19].

Exosomes are bi-lipid membrane vesicles that have a diameter of $50-100 \mathrm{~nm}$ and are secreted by various cell types. Exosomes carry a complex cargo load of proteins and RNAs that can potentially impact cellular and animal physiology. Studies have shown that exosomes are involved in complex physiological processes, such as intercellular communication, antigen presentation, and immune responses [20]. High-performance liquid chromatography (HPLC) and dynamic light scatter (DLS) analyses revealed that MSCs secrete cardioprotective microparticles with diameters ranging from 50 to $65 \mathrm{~nm}$ $[21,22]$. Furthermore, Lai et al reported that the therapeutic efficacy of exosomes derived from human embryonic stem cell-derived MSCs were similar to exosomes derived from other fetal tissue sources (e.g., the limbs and kidneys), demonstrating that MSC-derived exosomes display general therapeutic properties [23].

Silymarin, a milk thistle of Silybum marianum, is the well-researched drug in the treatment of liver disease [24]. It has been found that silymarin has hepatoprotective, anti-oxidant, anti-inflammatory activities [25]. Silymarin is in our study as a reference drug to compare the beneficial effects achieved by human menstrual blood-derived stem cell-derived exosomes (MenSC-Ex).

Because of these advantages, we sought to investigate the therapeutic potential of MenSC-Ex in D-GalN/LPSinduced FHF. There were two purposes to this study. First, we sought to investigate the therapeutic effects of MenSC-Ex on AML12 cells (in vitro) and D-GalN/LPSinduced FHF mice (in vivo). Second, we sought to identify the mechanism underlying the MenSC-Ex-mediated inhibition of liver apoptosis.

\section{Methods}

\section{Animals}

Six- to eight-week-old male C57BL/6 mice were purchased from Sippr-BK Laboratory Animal Corporation (Shanghai, China). The mice were fed food and water ad libitum and housed under standard conditions with a $12 \mathrm{~h}$ light and $12 \mathrm{~h}$ dark cycle. All animal experiments were approved by the Laboratory Animal Center of The
Tab of Animal Experimental Ethical Inspection of the First Affiliated Hospital, College of Medicine, Zhejiang University.

\section{Cell culture}

MenSCs were isolated and maintained as previously described $[4,12]$. The MenSCs were collected and cultured in Chang Medium (S-Evans Biosciences, Hangzhou, China). The MenSCs used in the experiments were at the fourth to eighth passage.

The mouse AML12 hepatocyte cell line was generously provided by the Stem Cell Bank of the Chinese Academy of Sciences. AML12 cells were cultured in DMEM/F12 (Gibco, Waltham, MA, USA) supplemented with $10 \%$ fetal bovine serum (FBS; Gibco), $100 \mathrm{IU} / \mathrm{mL}$ of penicillin (Sigma-Aldrich, St. Louis, MO, USA), $100 \mu \mathrm{g} /$ $\mathrm{mL}$ of streptomycin (Sigma-Aldrich), insulin, transferrin, selenium (ITS) Liquid Media Supplement (Sigma-Aldrich, USA) and $40 \mathrm{ng} / \mathrm{ml}$ dexamethasone (Sigma-Aldrich, USA).

\section{Identification of MenSCs using flow cytometry}

The expression of isolated MenSCs surface markers was evaluated using fluorescence-activated cell sorting (FACS). Briefly, $5 \times 10^{5}$ cells were collected and washed twice with stain buffer (BD Biosciences, San Jose, CA, USA). MenSCs were incubated in the dark for 20 min with the following primary antibodies: PE-conjugated CD29, CD34, CD45, CD73, CD90, CD105, CD117, and HLA-DR (Becton Dickinson, Franklin Lakes, NJ, USA). The stained cells were washed twice with stain buffer, resuspended in $500 \mu \mathrm{l}$ of stain buffer and then analyzed using a FC500 flow cytometer (Beckman Coulter, Brea, CA, USA). IgG1 (Becton Dickinson, Franklin Lakes, NJ, USA) was used as an isotype control for the anti-CD29, anti-CD34, antiCD45, anti-CD73, anti-CD90, anti-CD105, and antiCD117 antibodies. IgG2a (Becton Dickinson) was used as the isotype control for the anti-HLA-DR antibody. The results were analyzed using FlowJo software (Tree Star, Inc., Ashland, OR, USA).

\section{CFU-F assay}

The colony-forming unit-fibroblast (CFU-F) assay was determined as described previously [26]. MenSCs plated at 50,150 , or 250 cells per square centimeter. After 15 days of culture, cells were stained with $20 \%$ crystal violet solution for $15 \mathrm{~min}$ at room temperature. After phosphate-buffered saline (PBS) wash, the numbers of individual colonies were counted. Three independent experiments were performed.

\section{Isolation and identification of MenSC-Ex}

When MenSCs reached 70-80\% confluence, the cells were cultured for an additional $24 \mathrm{~h}$. The conditioned medium was collected and centrifuged at $2000 \mathrm{~g}$ for 
20 min to remove dead cells and cell debris. The supernatant was filtered through a $0.22-\mu \mathrm{m}$ pore filter (EMD Millipore, Billerica, MA, USA) and concentrated according to a $30 \mathrm{KDa}$ molecular weight cutoff (MWCO) (EMD Millipore) by centrifugation at $4000 \mathrm{~g}$ for $60 \mathrm{~min}$. A 1/5 volume of ExoQuick-TC Exosome Precipitation Solution (System Biosciences, Inc., Palo Alto, CA, USA) was added to the supernatant and it was incubated overnight. The mixture was centrifuged at $1500 \mathrm{~g}$ for 30 min, which aspirated the supernatant, and spun down at $1500 \mathrm{~g}$ for $5 \mathrm{~min}$ to remove residual ExoQuick-TC. The exosome-enriched fraction was diluted with $100 \mu \mathrm{l}$ PBS and stored at $-80^{\circ} \mathrm{C}$. The protein content of the concentrated exosomes was determined using a BCA protein assay kit (Pierce, Waltham, MA, USA). MenSC-Ex were identified using transmission electron microscopy. The MenSC-Ex were confirmed to express the exosome marker tetraspan molecules [CD63 (Abcam, Cambridge, UK) and tsg101 (Abcam)] using Western blot analysis.

\section{Cellular uptake and in vivo tracking of MenSC-Ex}

MenSC-Ex were labeled with a 1:10 ratio of Exo-Green (System Biosciences, Inc.). The exosomes solution was incubated at $37{ }^{\circ} \mathrm{C}$ for $10 \mathrm{~min}$. A $100 \mu \mathrm{l}$ volume of ExoQuick-TC was added to stop the labeling reaction. The labeled MenSC-Ex were placed on ice (or at $4{ }^{\circ} \mathrm{C}$ ) for $30 \mathrm{~min}$ and centrifuged for $3 \mathrm{~min}$ at 14,000 rpm to remove the supernatant, which contained excess label. The pellet containing the labeled MenSC-Ex was resuspended in $500 \mu \mathrm{l}$ of PBS, and at least $100 \mu \mathrm{l}$ of the solution of labeled MenSC-Ex was added to approximately $1 \times 10^{5}$ AML12 cells in one well of a 6-well culture plate, which was incubated for $24 \mathrm{~h}$. AML12 cells were incubated with $100 \mu \mathrm{l}$ of PBS instead of MenSC-Ex as the control. The cellular uptake of MenSC-Ex was observed under a confocal laser microscope (Carl Zeiss, Oberkochen, Germany).

XenoLight DiR (Perkin Elmer, Waltham, MA, USA) was used to track exosomes in vivo. The solution of XenoLight DiR was diluted to $300 \mu \mathrm{M}$ in PBS. We added the diluted XenoLight DiR solution to $10 \mu \mathrm{g}$ of MenSCEx in $1 \mathrm{ml}$ PBS to obtain a final concentration of $2 \mu \mathrm{M}$. The cells were incubated with MenSC-Ex at room temperature for $30 \mathrm{~min}$. A 1/5 volume of ExoQuick-TC Exosome Precipitation Solution (System Biosciences, Inc.) was then added to the supernatant for $30 \mathrm{~min}$. The solution was then centrifuged for $3 \mathrm{~min}$ at 14,000 rpm. The pellet, which contained the fluorescently labeled MenSC-Ex, was resuspended in $100 \mu \mathrm{l}$ of PBS. We then systemically administered $50 \mu \mathrm{g}$ of MenSC-Ex via the tail vein. IVIS analysis (Caliper Life Sciences, Hopkinton, MA, USA) and dissections were performed after $3 \mathrm{~h}$ and $6 \mathrm{~h}$.

\section{Antibody arrays}

Human Cytokine G1000 arrays (AAH-CYT-G1000; RayBiotech, Norcross, GA, USA) were used according to the manufacturer's instructions to measure the expression levels of 120 cytokines in the MenSCs and MenSC-Ex. Positive signals were captured on glass chips using a laser scanner (GenePix 4000B Microarray Scanner; Molecular Devices, Sunnyvale, CA, USA), and the observed fluorescence intensities were normalized to the intensities of the internal positive controls. These cytokines were screened using the following integrated conditions: the MenSC group compared to the MenSC-Ex group $(p<0.05)$ for samples with fluorescence intensity values that exceeded 300 (RayBiotech). Differentially expressed proteins were arranged using hierarchical clustering and represented as a heat map. The heat map was generated using R software (http://www.r-project.org/).

\section{Cell proliferation and apoptosis analysis}

A Cell Counting Kit-8 (CCK-8, Dojindo, Kumamoto, Japan) was used to evaluate proliferation in MenSC-Extreated D-GalN/LPS-induced AML12 cells. After the cells were cultured for $24 \mathrm{~h}$ with $44 \mu \mathrm{g} / \mathrm{ml} \mathrm{D-GalN}$ and $100 \mathrm{ng} / \mathrm{ml} \mathrm{LPS}$, the CCK- 8 reagent was added to the chamber, and the cells were incubated for an additional $3 \mathrm{~h}$ according to the manufacturer's protocol. The optical densities of the solutions were read at $450 \mathrm{~nm}$ (OD450) and measured using a multifunctional microplate reader (SpectraMax M5, Molecular Devices).

To analyze cell apoptosis, MenSC-Ex-treated AML12 cells that were cultured for $24 \mathrm{~h}$ with $44 \mu \mathrm{g} / \mathrm{ml} \mathrm{D}$-GalN and $100 \mathrm{ng} / \mathrm{ml}$ LPS were collected, centrifuged, and then stained with propidium iodide and Annexin $\mathrm{V}$ in the dark for $30 \mathrm{~min}$ at room temperature using a cell apoptosis Analysis Kit (Sigma-Aldrich). Cell apoptosis was then analyzed using flow cytometry.

\section{The animal model and MenSC-Ex transplantations}

To induce FHF in mice, C57BL/6 mice $(20 \pm 2$ g) were intraperitoneally injected with D-GalN $(800 \mathrm{mg} / \mathrm{kg})$ (Sigma-Aldrich) and LPS (50 $\mu \mathrm{g} / \mathrm{kg}$ ) (Sigma-Aldrich). Mice injected with an equal volume of PBS alone were used as the model group for the FHF model $(n=10$ per model group). To evaluate the therapeutic efficacy of MenSC-Ex on FHF, $1 \mu \mathrm{g} / \mu \mathrm{l}$ of MenSC-Ex in PBS or PBS alone was injected into the tail veins of mice 1 day before treatment. The animals were anesthetized using sodium pentobarbital $(50 \mathrm{mg} / \mathrm{kg}$; Solarbio Bioscience \& Technology, Shanghai, China) 6 hours after treatment with D-GalN/LPS. The serum samples were centrifuged at $3000 \mathrm{rpm}$ for $10 \mathrm{~min}$ to collect clear serum to detect the levels of alanine aminotransferase (ALT) and aspartate aminotransferase (AST). A portion of liver tissue was stored in $4 \%$ paraformaldehyde for histological and 
immunohistochemical analysis. The remainder of the tissue samples was washed in cold saline and preserved at $-80{ }^{\circ} \mathrm{C}$ for further analysis using reverse transcriptionpolymerase chain reaction (RT-PCR). The survival rates of the mice were determined for the $12 \mathrm{~h}$ period following D-GalN/LPS challenge.

\section{Liver function tests and ELISA}

Liver function was assessed by analyzing serum alanine aminotransferase (ALT) and aspartate aminotransferase (AST) levels. ALT and AST levels were measured using commercial kits (Nanjing Jiancheng Bioengineering Institute, Jiangsu, China) according to the manufacturer's instructions.

The levels of interleukin-6 (IL-6), interleukin-1 $\beta$ (IL-1 $\beta$ ), and tumor necrosis alpha (TNF- $\alpha$ ) were measured in serum samples using commercially available enzyme-linked immunosorbent assays (ELISAs) (RayBiotech) according to the manufacturer's instructions.

\section{Histological, immunohistochemistry and TUNEL staining}

Liver tissues were harvested from mice $6 \mathrm{~h}$ after treatment with D-GalN/LPS or MenSC-Ex. Tissues were fixed in $10 \%$ buffered formalin, embedded in paraffin, sectioned to a 5-mm thickness, and stained with hematoxylin and eosin (H\&E). The sections were imaged using an Olympus IX83 inverted microscope (Olympus, Tokyo, Japan) equipped with Olympus cellSens software (cellSens Standard 1.9).

To perform the immunohistochemistry test, peroxidase activity was blocked by incubating the sections in 3\% $\mathrm{H}_{2} \mathrm{O}_{2}$ for 10 min. The sections were then pretreated via heat-mediated antigen retrieval with sodium citrate buffer (pH6.0). The tissue sections were blocked in 10\% FBS for 20 min at room temperature and then incubated with rabbit polyclonal antibodies against mouse caspase3 (Cell Signaling Technology, Danvers, MA, USA) overnight at $4{ }^{\circ} \mathrm{C}$. The slides were washed three times with PBS for 5 min and subsequently incubated with secondary antibodies (Abcam). A solution of diaminobenzidine tetrahydrochloride (DAB kit; Maixin Biotech, Fujian, China) was used as the reaction substrate.

Apoptosis was detected in liver cells in paraffinembedded sections using a fluorescence terminal deoxynucleotidyl transferase dUTP nick-end labeling (TUNEL) apoptosis assay kit (Vazyme, Nanjing, China) according to the manufacturer's instructions. Stained sections were observed and photographed using an Olympus IX83 inverted microscope equipped with Olympus cellSens software. The number of positive cells was counted in six randomly selected fields per slide.

\section{DNA fragmentation analysis}

Genomic DNA was extracted from liver samples according to the instructions supplied by the manufacturer of the DNA Ladder kit (Beyotime, Jiangsu, China). The DNA was then electrophoresed in a 1.5\% agarose gel, which was stained with $0.1 \mathrm{~g} / \mathrm{ml}$ ethidium bromide at $140 \mathrm{~V}$ for $20 \mathrm{~min}$.

\section{Isolation of liver mononuclear cells}

Liver mononuclear cells (MNCs) were isolated and prepared as previously described [27]. Briefly, the livers of C57BL/6 mice were passed through a $70-\mu \mathrm{m}$ stainless steel mesh. The precipitated cells were resuspended in 40\% Percoll (Sigma-Aldrich), gently lain over 70\% Percoll and centrifuged at $2000 \mathrm{~g}$ for $20 \mathrm{~min}$ at room temperature. The MNCs were contained in and isolated from the interphase. Liver MNCs were stained using anti-mouse CD11b, CD3, NK1.1, and F4/80 antibodies (Biolegend, San Diego, CA, USA) and subjected to FACS analysis.

\section{Quantitative real-time RT-PCR}

Quantitative real-time RT-PCR (qRT-PCR) was used to analyze mRNA expression levels using a CFX96 Real-time PCR Detection System (Bio-Rad, Hercules, CA, USA). These experiments were performed according to a previously described protocol [28]. Briefly, the reaction consisted of $1 \mu \mathrm{L}$ of cDNA, $8.2 \mu \mathrm{L}$ of RNAse-free water, $10 \mu \mathrm{L}$ of SYBR $^{\circ}$ Fast qPCR Master Mix (Takara Bio Inc., Mountain View, CA, USA), and $0.4 \mu \mathrm{L}$ of each gene-specific primer $(10 \mathrm{mM})$. The primer sequences are shown in Table 1 . The relative quantities of each $\mathrm{PCR}$ product were determined using the following equation: $R Q=2^{-\triangle \Delta C T}$. GAPDH served as an internal control.

\section{Western blot analysis}

After MenSCs were collected, the cells and MenSC-Ex were homogenized using cell lysis buffer (Cell Signaling Technology) containing 100× phenylmethylsulfonyl fluoride (PMSF) (Beyotime Biotechnology Inc., Shanghai, China).

Table 1 Primers used for qRT-PCR analysis

\begin{tabular}{|c|c|c|}
\hline Primer name & Sequence $\left(5^{\prime}-3^{\prime}\right)$ & Species \\
\hline GAPDH-F & AATGGATTTGGACGCATTGGT & Mouse \\
\hline GAPDH-R & TTTGCACTGGTACGTGTTGAT & \\
\hline IL-6-F & GGCGGATCGGATGTTGTGAT & Mouse \\
\hline IL-6-R & GGACCCCAGACAATCGGTTG & \\
\hline IL-1 $\beta-F$ & GTACATCAGCACCTCACAAG & Mouse \\
\hline IL-1 $\beta-R$ & CACAGGCTCTCTTTGAACAG & \\
\hline TNF-a-F & ACTCCCAGAAAAGCAAGCAA & Mouse \\
\hline TNF-a-R & CGAGCAGGAATGAGAAGAGG & \\
\hline Caspase-3-F & TACCGGTGGAGGCTGACT & Mouse \\
\hline Caspase-3-R & GCTGCAAAGGGACTGGAT & \\
\hline
\end{tabular}


Twenty micrograms of total protein were obtained from MenSCs and MenSC-Ex and placed in separate lanes to be separated using electrophoresis on NuPAGE ${ }^{\circ}$ Novex $10 \%$ Bis-Tris gels (Life Technologies, Carlsbad, CA, USA). The separated proteins were then transferred onto PVDF membranes (EMD Millipore). The membranes were blocked in $0.5 \%$ bovine serum albumin (BSA) for $1 \mathrm{~h}$ at room temperature and then incubated with primary antibodies at $4{ }^{\circ} \mathrm{C}$ overnight. They were subsequently incubated with HRP-conjugated secondary antibodies (goat anti-mouse or goat anti-rabbit, Bio-Rad) for $1 \mathrm{~h}$ at room temperature. Immunoreactive bands were visualized using enhanced enhanced chemiluminescence (ECL) reagent (Bio-Rad) with a Tanon-4500 digital image system (Tanon Science \& Technology, Shanghai, China).

\section{Statistical analysis}

All statistical analyses were performed using GraphPad Prism v5.0 (GraphPad Software Inc., San Diego, CA, USA). All data represent the means \pm standard deviation. Oneway analysis of variance (ANOVA) was used to determine differences between groups. $P$ values $<0.05\left(^{*}\right)$ or $<0.01\left({ }^{* * *}\right)$ were considered to indicate statistical significance.

\section{Results}

\section{Identification of MenSCs}

MenSCs have morphologies and immunophenotypes that are similar to MSCs. The MenSCs exhibited a spindleshaped, fibroblast-like morphology (Fig. 1A); and they expressed high levels of CD29, CD73, CD90, and CD105 and did not express CD34, CD45, CD117, or HLA-DR
A
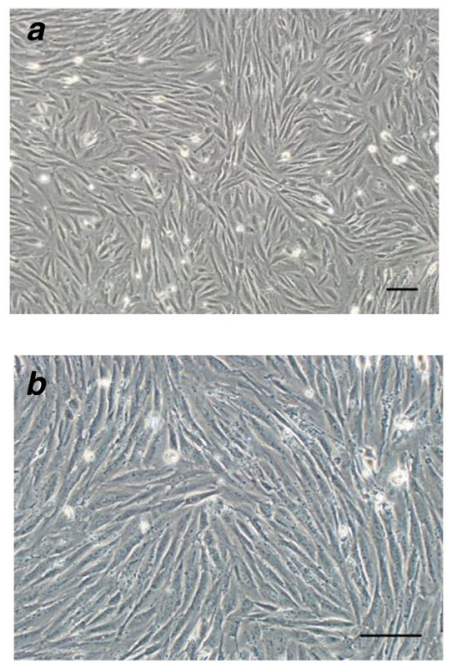

B
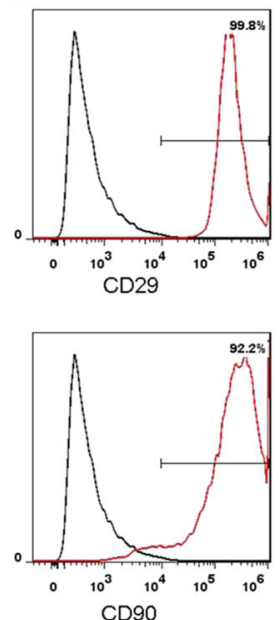
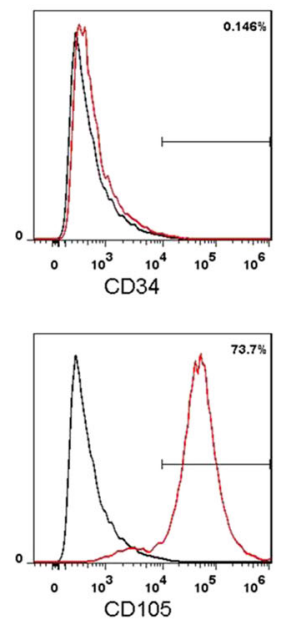

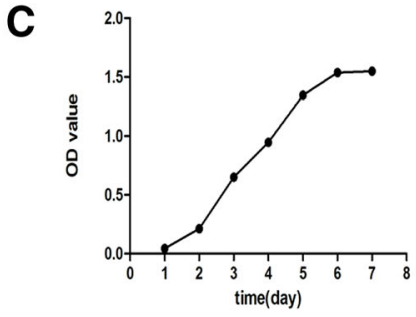

D

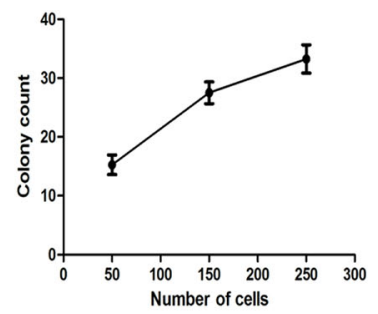

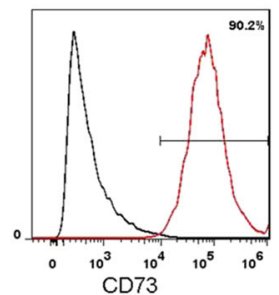
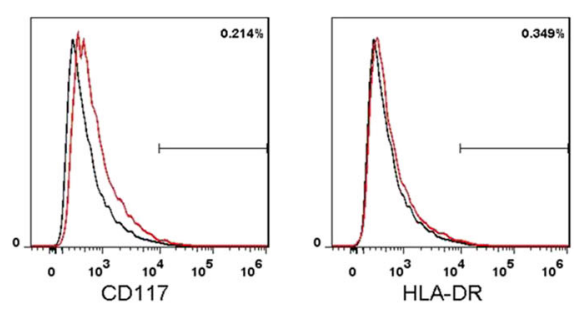

Fig. 1 Identification of MenSCs. A Representative images of MenSCs shown at (a) scale bar = $100 \mu \mathrm{m}$ and (b) scale bar $=100 \mu \mathrm{m}$. B Flow cytometry analysis of the surface markers expressed on MenSCs. C Growth curve of MenSCs by CCK-8 assay. D Colony count of MenSCs after 15 days of culture at 50, 150, and 250 cells per square centimeter 
(Fig. 1B). MenSCs exhibited a higher proliferation rate (Fig. 1C) and displayed enhanced colony-forming (CFU-F) ability depending on the number of cells (Fig. 1D).

\section{Identification of MenSC-Ex}

MenSC-Ex was prepared as previously described. Transmission electron microscopy (TEM) showed that MenSCEx displayed a round, ball-like shape and had diameters of approximately 30-100 nm (Fig. 2A). Western blot analysis showed that the collected MenSC-Ex expressed specific exosomal surface markers, such as CD63 and tsg101, which are not expressed on MenSCs (Fig. 2B). These results demonstrate that MenSC-Ex display specific characteristics that are identical to those described in previous studies of exosomes [29, 30].

Differential cytokine expression in MenSCs and MenSC-Ex To identify molecules expressed on MenSCs and MenSCEx, an antibody array was used to examine cytokine levels (Fig. 3A). Several cytokines, including intercellular cell adhesion molecule-1 (ICAM-1), angiopoietin-2, Axl, angiogenin, insulin-like growth factor-binding protein 6 (IGFBP-6), osteoprotegerin, IL-6 and IL-8, were expressed at higher levels on MenSC-Ex than on MenSCs. Among the 120 cytokines that were evaluated in this array, some were not expressed at detectable levels or were expressed at extremely low levels (data not shown). The cytokines that were differentially expressed are displayed in a heat map (Fig. 3B). The average fluorescence intensities associated with each marker are shown in Fig. 3C.

\section{MenSC-Ex were taken up by AML12 cells in vitro and tracked in mice in vivo}

To determine whether MenSC-Ex can be taken up by AML12 cells, we labeled MenSC-Ex with Exo-Green, a fluorescent cell linker compound that is incorporated into cellular proteins. When we incubated the ExoGreen labeled exosomes with AML12 cells, subsequently green fluorescence in the cytoplasm of almost every AML12 cell was observed (Fig. 4A). These results indicated that a significant number of exosomes were taken up by the AML12 cells in vitro.

To determine whether MenSC-Ex can be tracked in C57/BL6 mice, MenSC-Ex were labeled with XenoLight DiR. We administered the DiR-labeled MenSC-Ex into the tail veins of C57/BL6 mice, and then evaluated their distribution using in vivo imaging. At $3 \mathrm{~h}$ and $6 \mathrm{~h}$ after the injection, fluorescence was detected in the liver, the lungs, and the spleen (Fig. 4B). The in vivo fluorescence intensity indicated that the signal was retained in the liver, the lungs, and the spleen at a steady level after $3 \mathrm{~h}$ and $6 \mathrm{~h}$. We also intravenously injected PBS as a control.

\section{MenSC-Ex inhibited apoptosis in D-GaIN/LPS-induced AML12 cells}

To measure the effect of D-GalN/LPS on the viability of AML12 cells, the ratio of its inhibitory effect was determined using a CCK-8 assay. Three different doses $(5 \mu \mathrm{g}$, $10 \mu \mathrm{g}$, and $20 \mu \mathrm{g}$ ) of MenSC-Ex were found to inhibit the effects induced by D-GalN/LPS on AML12 cells. The data for five of the groups showed significant differences. Furthermore, the inhibitory ratio increased in a dose-dependent manner as the dose of MenSC-Ex increased. These results demonstrated that MenSC-Ex exert an anti-apoptosis effect on AML12 cells (Fig. 5A).

To determine the effects of MenSC-Ex on D-GalN/ LPS-induced AML12 apoptosis, AML12 cells were pretreated with either exosomes or PBS (both in FBS-free medium) for $24 \mathrm{~h}$. The cells were then co-incubated with D-GalN/LPS for an additional $6 \mathrm{~h}$. Apoptosis was measured in the AML12 cells using Annexin V/PI (Fig. 5B). When cells were treated with D-GalN/LPS, the
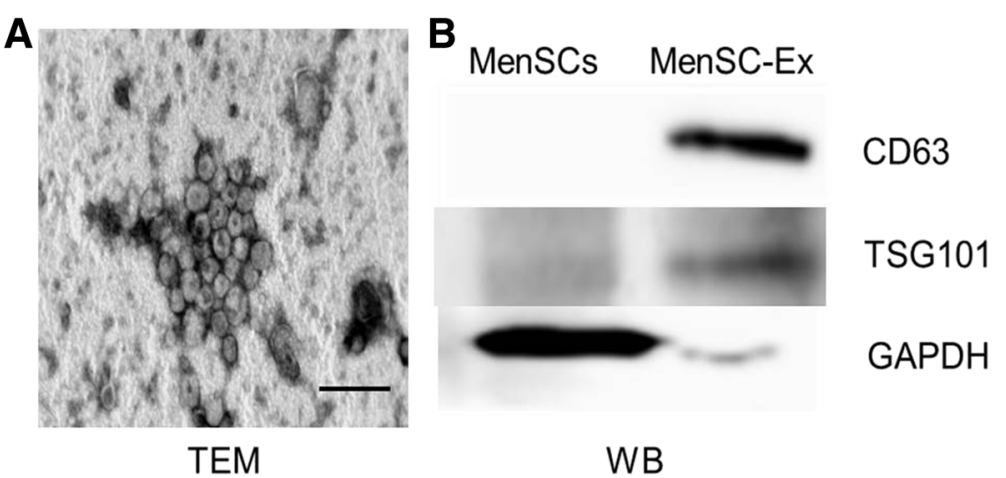

Fig. 2 Identification of MenSC-Ex. A Transmission electron micrograph (TEM) of MenSC-Ex, scale bar = $200 \mathrm{~nm}$. B CD63 and tsg101 were detected in MenSC-Ex using Western blot analysis 

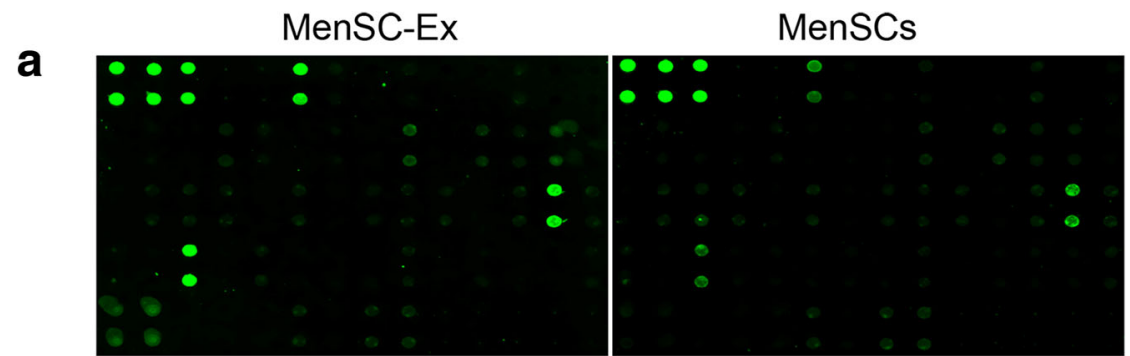

\section{0
1
$\frac{1}{2}$
0
1
$\frac{1}{2}$}
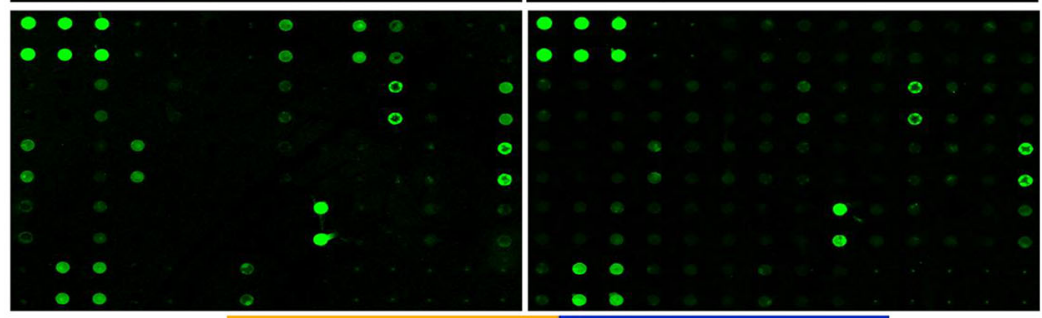

\begin{tabular}{l}
0 \\
1 \\
1 \\
\hline \\
1 \\
1 \\
$\frac{1}{4}$
\end{tabular}

b

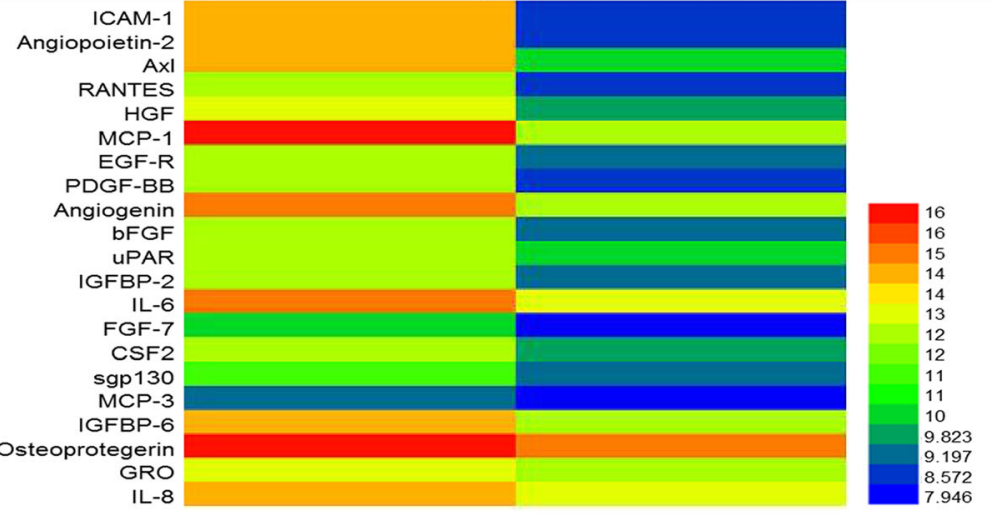

C

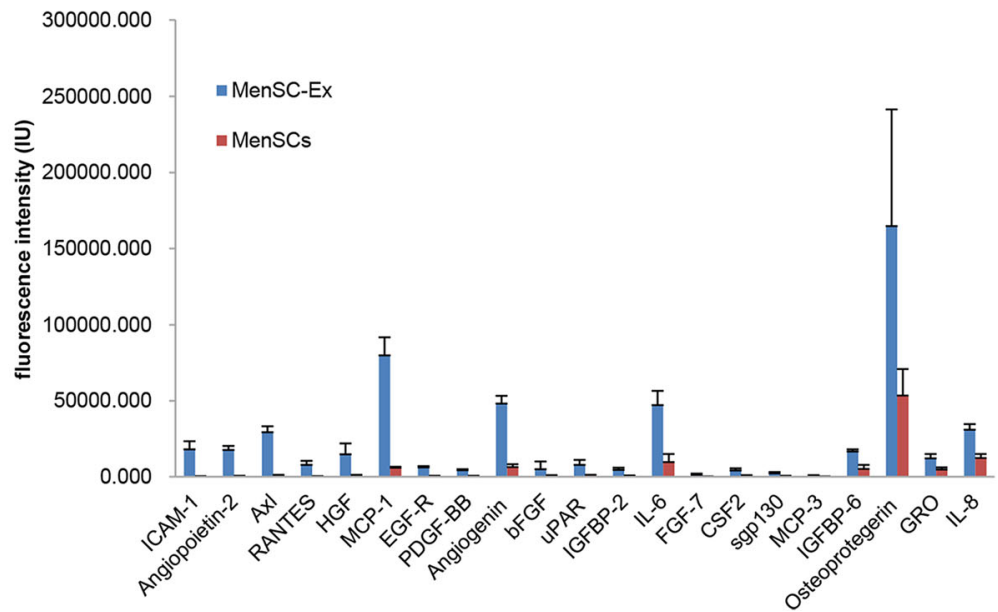

Fig. 3 Cytokine expression in MenSC-Ex and MenSCs. A Representative array images are shown $(n=4)$. B Cytokines that were differentially expressed are shown as a heat map. C The fluorescence intensities of the indicated cytokines

ratio of cells undergoing apoptosis in the AML12 cells was higher than in the MenSC-Ex-treated group. There were significant differences in the proportions of apoptotic cells between the three different doses of MenSCEx (Fig. 5C). These results suggest that MenSC-Ex inhibit D-GalN/LPS-induced apoptosis in AML12 cells.
MenSC-Ex enhanced the survival rate and improved liver function in a D-GalN/LPS-induced mouse model of FHF Mice began to die at $6 \mathrm{~h}$ after they were injected with D-GalN/LPS, and the mortality rate in these mice reached $80 \%$ within $12 \mathrm{~h}$. However, mice pretreated with MenSC-Ex had significantly reduced mortality (Fig. 6A). 

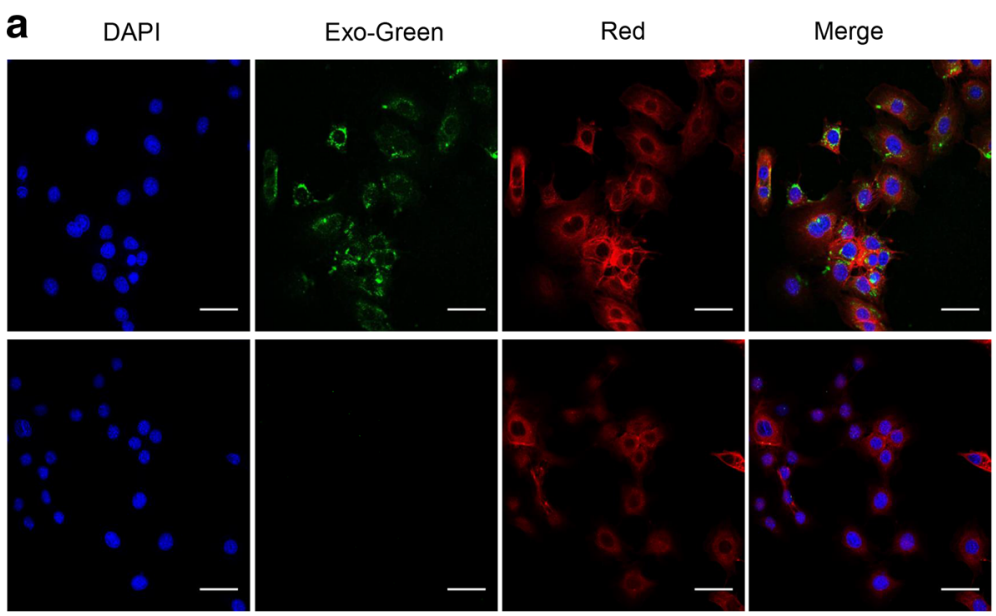

DAPI

PBS

Red

Merge

b

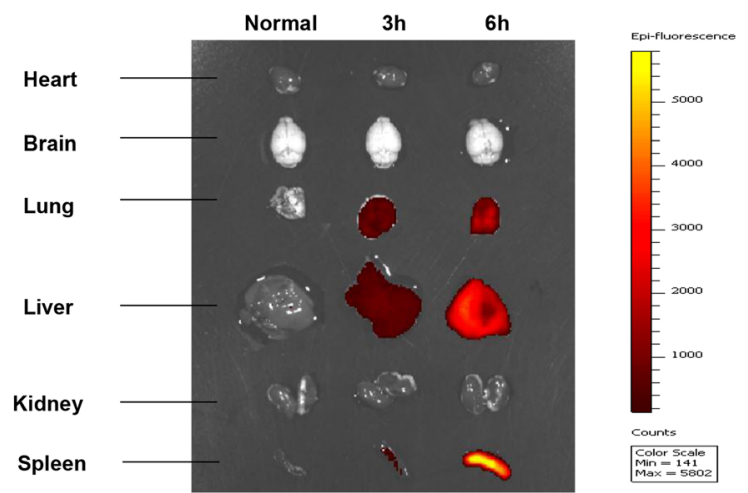

Fig. 4 Uptake and tracking of MenSC-Ex in AML12 cells and mice. A Intracellular Exo-Green-labeled exosomes were detected in AML12 cells using confocal fluorescence microscopy. Scale bar $=50 \mu \mathrm{m}$ (B). Analysis of XenoLight DiR-labeled exosomes after systemic administration was detected using an in vivo imaging system (IVIS)

Mice treated with silymarin, the positive control, exhibited a lower protective effect than was observed in the mice treated with MenSC-Ex.

D-GalN/LPS increased the levels of ALT and AST in the serum of C57/BL6 mice, resulting in a significant increase in liver injury over the level observed in the PBS group, the silymarin-treated group, and the MenSC-Extreated group. Mice pretreated with MenSC-Ex showed significantly lower levels of ALT and AST than were observed in the group treated with D-GalN/LPS alone $(p<0.01)$. There was no significant difference between the MenSC-Ex group and the silymarin group (Fig. 6B, C).

In the FHF mouse model, real-time PCR data confirmed that MenSC-Ex significantly downregulated hepatic levels of TNF- $\alpha$, IL- 6 , and IL- $1 \beta$, suggesting that MenSC-Ex prevented D-GalN/LPS-induced FHF by inhibiting the production of inflammatory cytokines $(p<0.01)$ (Fig. 6D-F). Furthermore, the results of ELISA showed that D-GalN/LPS increased the levels of the inflammatory cytokines TNF- $\alpha$, IL- 6 , and IL- $1 \beta$ in C57/BL6 mouse serum. However, treatment with MenSC-Ex resulted in significantly lower levels of TNF- $\alpha$, IL- 6 , and IL- $1 \beta$ than were observed in the DGalN/LPS-induced mice $(p<0.01)$ (Fig. 6F-I).

To assess general morphological changes in the liver, liver tissue sections were mounted and stained with H\&E (Fig. 6J). The normal groups showed a normal liver architecture. The groups administered with D-GalN/LPS displayed severe centrilobular focal necrosis, apoptosis and inflammation. Mice pretreated with MenSC-Ex and then induced with D-GalN/LPS showed a much lower degree of hepatocellular necrosis and inflammation than were observed in the D-GalN/LPS group.

MenSC-Ex inhibited apoptosis in hepatocytes and the expression of caspase-3 in D-GalN/LPS-induced FHF Apoptosis was detected in hepatocytes using fluorescent TUNEL staining. A large number of TUNEL-positive 


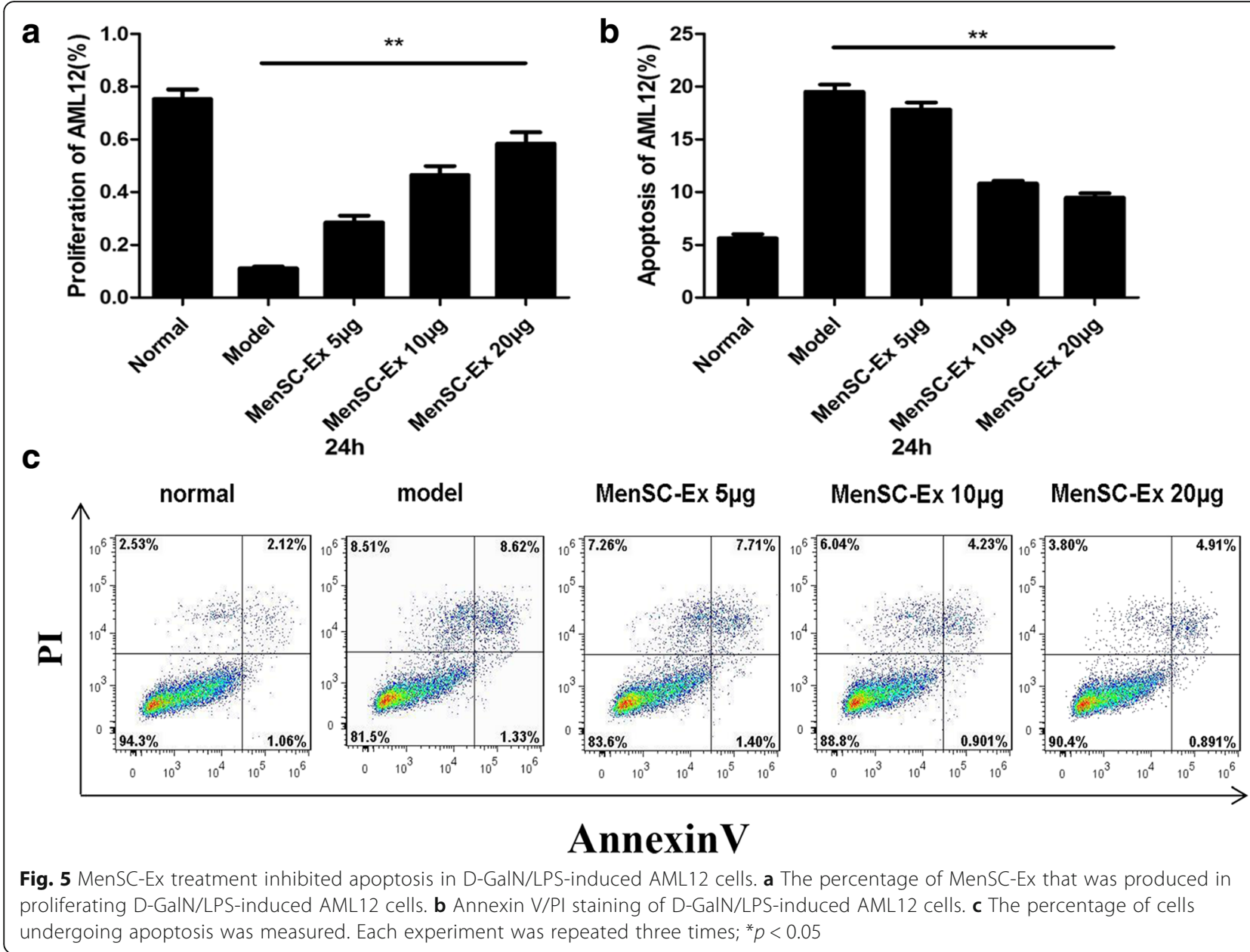

hepatocytes were observed in liver tissues obtained from mice treated with D-GalN/LPS. However, few TUNEL-positive hepatocytes were observed in the livers of mice in the MenSC-Ex and silymarin groups. There were substantial differences in the number of apoptotic hepatocytes between the D-GalN/LPS and MenSC-Ex groups (Fig. 7A).

Genomic DNA fragmentation was assayed to confirm that hepatocytes were undergoing apoptosis. DNA fragmentation was observed in the livers of mice treated with D-GalN/LPS, while no DNA fragmentation was observed in the livers of the MenSC-Ex group, and only a small amount of DNA fragmentation was observed in the silymarin group (Fig. 7B).

Apoptosis is a physiological process that is involved in D-GalN/LPS-induced FHF. At $6 \mathrm{~h}$ after MenSC-Ex transplantation, immunohistochemistry and PCR for caspase- 3 showed that there were caspase-3-positive cells in the MenSC-Ex livers $(p<0.01)$, the PBS and silymarin livers exhibited lower levels than the DGalN/LPS group (Fig. 7C, D).
MenSC-Ex inhibited macrophage proliferation in liver mononuclear leukocytes in D-GaIN/LPS-induced FHF The percentage of natural killer (NK) cells that were in the liver was calculated by multiplying the percentage of $\mathrm{CD}^{-}{ }^{-} \mathrm{NK} 1.1^{+}(\mathrm{NK})$ cells by the total number of lymphocytes per liver. NK cells $\left(\mathrm{CD}^{-} \mathrm{NK} 1.1^{+}\right)$and total T cells $\left(\mathrm{CD}^{+} \mathrm{NK} 1.1^{-}\right)$were also detected in the liver using flow cytometry. The results showed that treatment with DGalN/LPS induced NK cells to accumulate in the liver $(p<0.01)$ (Fig. 8A, C). Administration of MenSC-Ex significantly prevented liver injury. There was no significant difference between the percentage of NK cells in the MenSC-Ex and silymarin groups. Furthermore, the severe liver injury that was triggered in the C57/BL6 mice that were treated with D-GalN/LPS indicated that NK cells could potentially mediate D-GalN/LPS-induced FHF.

Flow cytometry analysis was used to detect mononuclear leukocytes in the livers of D-GalN/LPS-induced FHF mice. CD11b is a marker of mononuclear leukocytes, and F4/80 is a specific marker of macrophages, including Kupffer cells. In normal mice, the percentage of 

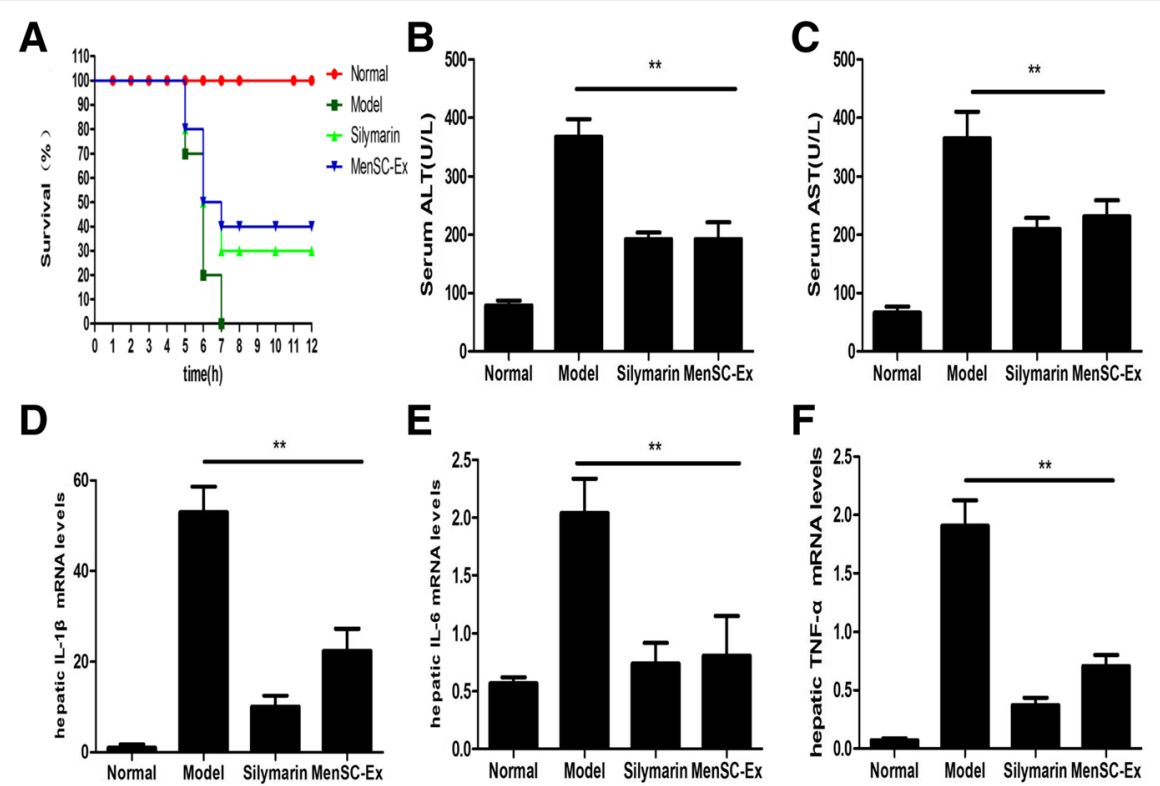

E

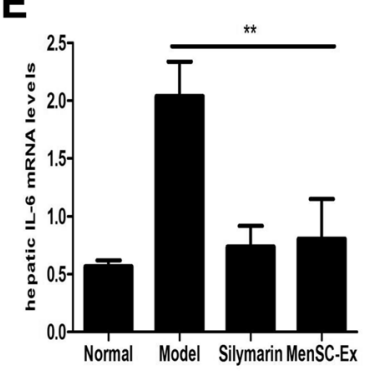

$\mathbf{F}$

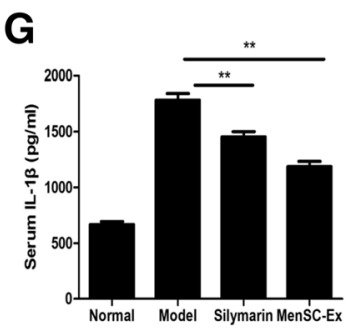

H
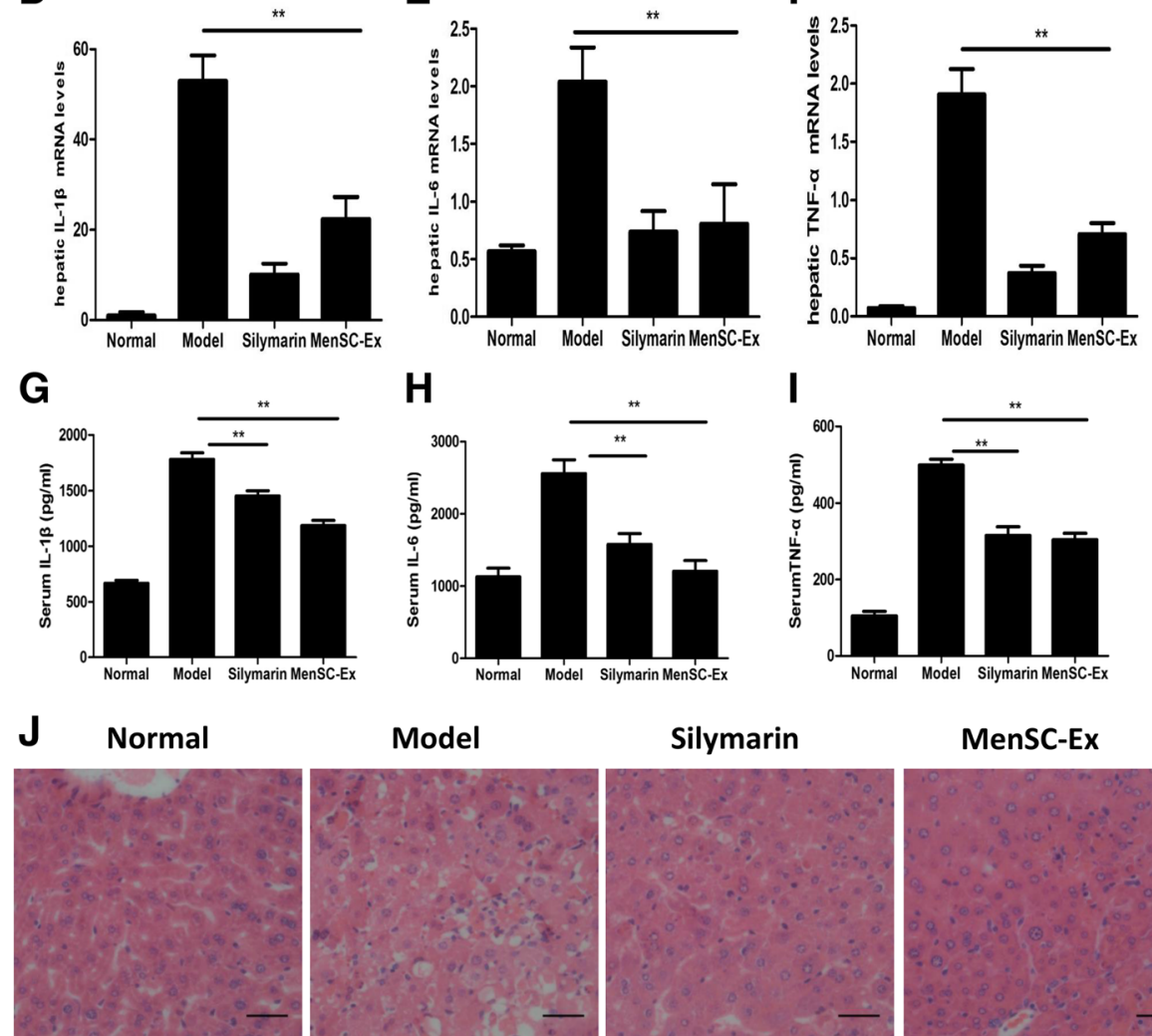

Model

Silymarin

MenSC-Ex
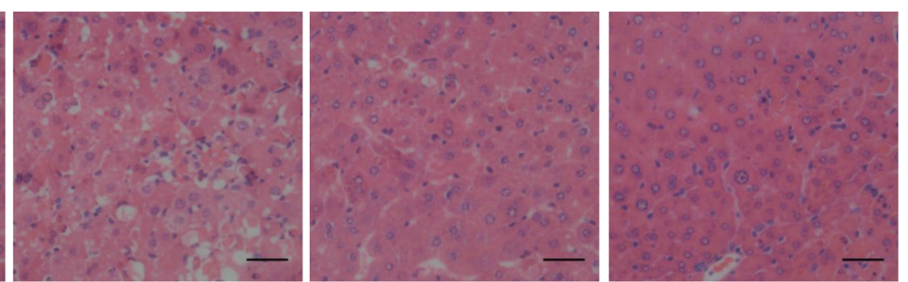

Fig. 6 MenSC-Ex transplantation enhanced survival rates and improved liver function in a D-GalN/LPS-induced FHF mouse model. a Survival curves after mice were injected with D-GalN/LPS $(n=10)$. $\mathbf{b}$ and $\mathbf{c}$ Serum levels of ALT and AST were measured at $6 \mathrm{~h}$ after stimulation with D-GalN/LPS. $\mathbf{d}$-f Liver levels of the IL-6, IL-1 $\beta$, and TNF-a mRNAs were also detected using real-time RT-PCR at $6 \mathrm{~h}$ after the nice were injected with D-GalN/LPS. g-i Serum levels of IL-6, IL-1 $\beta$, and TNF-a were determined using ELISA. $\mathbf{j}$ Liver sections were obtained from D-GalN/LPS-induced mice and analyzed using H\&E staining $\left(n=10\right.$ per group, $\left.{ }^{* *} p<0.01\right)$. Scale bar $=50 \mu \mathrm{m}$

$\mathrm{CD} 11 \mathrm{~b}+$ cells in the livers was $0.7 \%$. After mice were stimulated with D-GalN/LPS, this percentage significantly increased to $26.7 \%$. However, it decreased to $11.7 \%$ after the mice were treated with MenSC-Ex $(p<0.01)$ (Fig. 8B, $D)$. There was no significant difference in the percentage of CD11b + cells between the MenSC-Ex and silymarin groups. These results demonstrated that MenSC-Ex inhibits the recruitment of inflammatory cells and reduces the number of inflammatory cells in liver. However, there were no significant differences in the numbers of $\mathrm{CD} 11 \mathrm{~b}+/ \mathrm{F} 4 / 80+$ cells among all four groups.

\section{Discussion}

Because they are easy to collect, isolate, and there are no ethical considerations associated with their use, MenSCs have become a useful tool for exploring how MSCs can be used to treat tissue injuries. Our group previously reported that MenSCs are a promising therapeutic method for treating some diseases, such as liver injury and type 1 diabetes [12, 31]. Based on these benefits, we chose to use MenSCs as a source of stem-cell-derived exosomes. In this study, MenSC-Ex were used in mice, and no evidence of immune rejection was observed when MenSC- 


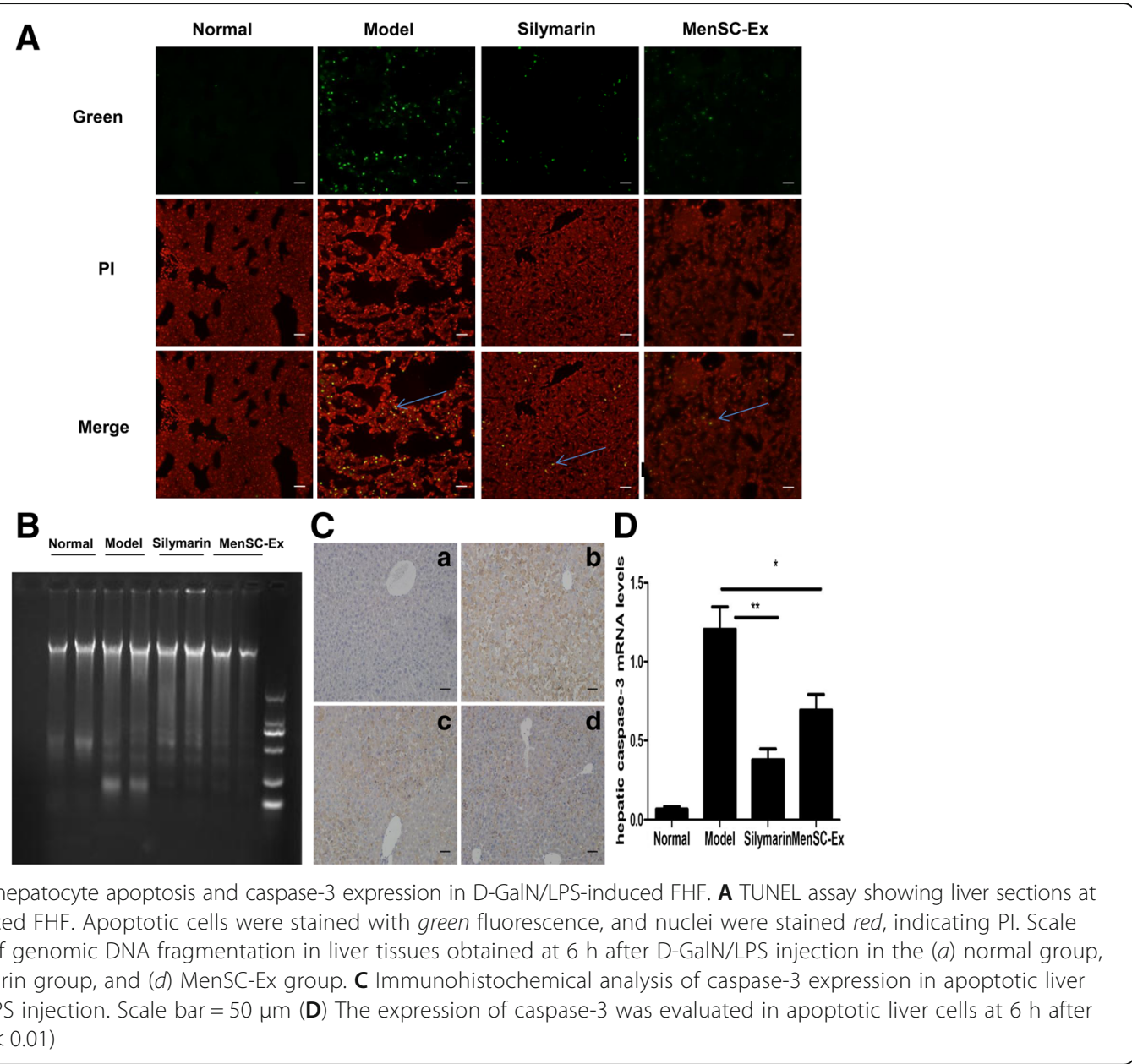

Ex were transplanted into mice. We successfully isolated exosomes from MenSCs. The MenSC-Ex had diameters of approximately 30-100 $\mathrm{nm}$ and expressed the CD63 and tsg101 proteins.

Several articles have shown that MSCs may perform paracrine functions that might play therapeutic roles in disease states [32-35]. It has been reported that human bone marrow MSC-derived microvesicles contain RNAs and surface proteins that had therapeutic effects in a mouse model of acute tubular injury, in which they enhanced survival [36-38]. In the present study, we used an antibody array to show that MenSC-Ex contain many cytokines, including ICAM-1, angiopoietin-2, Axl, angiogenin, IGFBP-6, osteoprotegerin, IL-6, and IL-8. Generally, IL-6 has been known to act as a pro-survival factor, which can stimulate hepatocyte proliferation [39, 40]. Osteoprotegerin could regulate bone resorption, modeling, and remodeling, which is mainly secreted by osteoblasts [41, 42]. Meanwhile, IGFBP- 6 could control the differentiation of cells and prevent the apoptosis and aging of human fibroblasts $[43,44]$. IL-8, a chemokine of the immune system, could recruit neutrophils, endothelial cells, and macrophages to sites of injured tissues $[45,46]$. Recently, Ren et al. found that MSCs express a low level of ICAM-1, which play a critical role in MSC-mediated immunosuppression [47]. Axl is a transmembrane receptor tyrosine kinase, expressed widely in the body, which could regulate apoptosis, migration and proliferation of cells through a variety of signaling pathways [48]. According to Cai et al., angiopoietin-2 could promote the generation of new vessels in retina under hypoxia [49]. Angiogenin is capable of stimulating cell proliferation and promoting cell survival, which is predominately produced by the liver and normally circulating in human body fluid [50]. Therefore, all of the cytokines that we found were expressed in MenSC-Ex may have potentially therapeutic effects on diseases.

We evaluated liver cells in vivo and in vitro using fluorescence-labeled MenSC-Ex. Experiments in which treated cells or mice with MenSC-Ex provided initial insights into potential cellular therapies that might involve these structures. We observed clear fluorescence in AML12 cells and mice post-treatment with fluorescence-labeled MenSC-Ex. These data suggest that MenSC-derived 


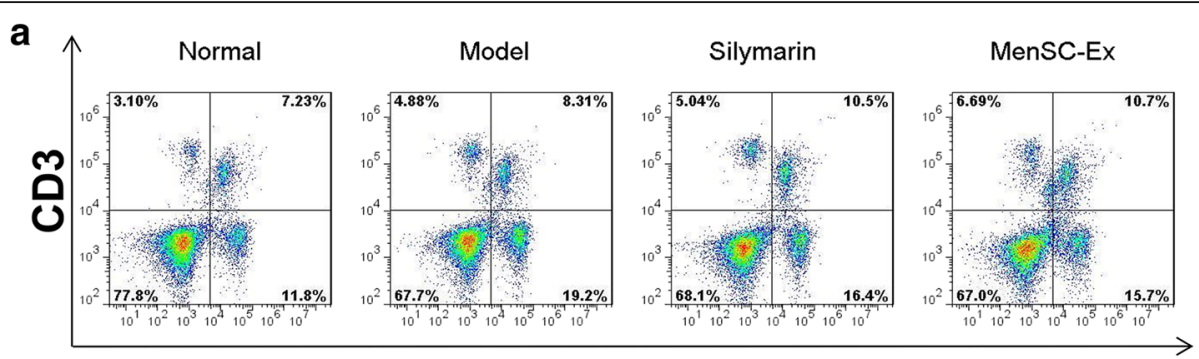

NK1.1

b

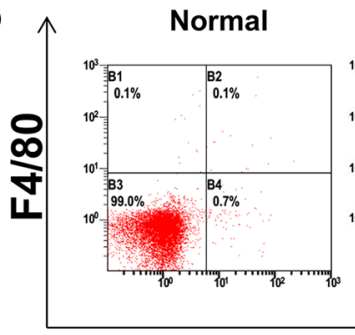

Model

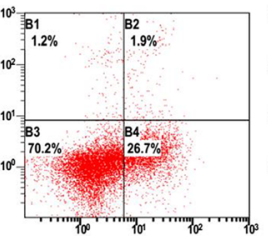

Silymarin

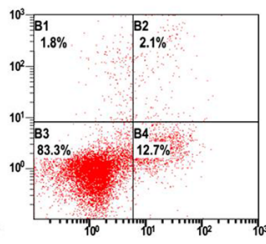

MenSC-Ex

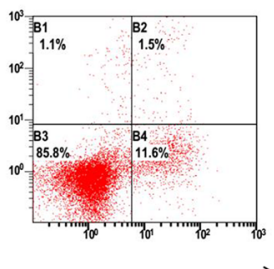

\section{CD11b}
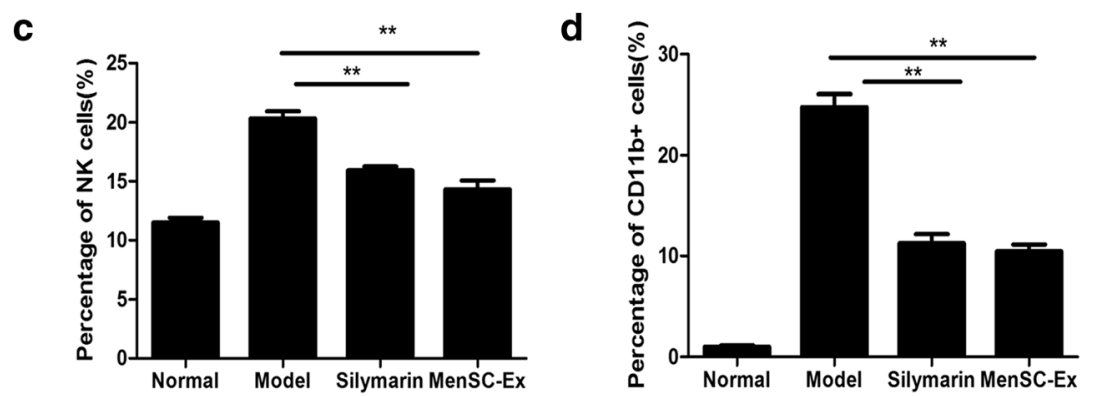

Fig. 8 MenSC-Ex inhibited proliferation in macrophages in cultures of liver mononuclear leukocytes in D-GalN/LPS-induced FHF. a Mononuclear leukocytes obtained from the liver were co-stained with anti-mouse CD3 and NK1.1 antibodies at $6 \mathrm{~h}$ after D-GalN/LPS-induced FHF. b Mononuclear leukocytes in the liver were co-stained with anti-mouse CD11b and F4/80 antibodies. $\mathbf{c}$ The percentages of NK cells $\left(\mathrm{CD}^{-} \mathrm{NK} 1.1^{+}\right)$in the total population of mononuclear leukocytes are shown $\left({ }^{*} p<0.01\right)$. $\mathbf{d}$ The percentages of $C D 11 b^{+}$cells out of the total population of mononuclear leukocytes are shown $\left.{ }^{* *} p<0.01\right)$

exosomes are capable of entering into and performing functions within injured livers.

We used a D-GalN/LPS-induced mouse model of FHF to explore the mechanisms underlying clinical liver failure. In the present study, administering an injection containing MenSC-Ex prior to D-GalN/LPS reduced the circulating levels of TNF- $\alpha$, IL-6, and IL- $1 \beta$, decreased hepatic apoptosis, improved liver function, and eventually lowered the fatality rate in FHF mice. Our findings also demonstrated that MenSC-Ex inhibited hepatocyte apoptosis and enhanced survival in mice treated with DGalN/LPS. Specifically, an intravenous injection of MenSC-Ex reversed organ failure. All of our results showed that MenSC-Ex functioned to affect important pharmacological characteristics.

According to the results of our experiments, the mechanisms that explain the therapeutic effect of MenSC-Ex on D-GalN/LPS-induced FHF involves the inhibitory immunomodulation of activated MNC proliferation or the activation of apoptosis-associated proteins. In the present study, one dataset suggested that there were more MNC in the D-GalN/LPS group than in the group treated with MenSC-Ex and the positive control group. One possible explanation for this result is that, in vivo MenSC-Ex may inhibit MNC proliferation, and MenSC-Ex may thereby act as crucial contributors to the observed reductions in FHF. The amount of cleaved caspase-3 expressed in the D-GalN/LPS group was lower than the amount expressed in the MenSC-Ex, but there was no difference with the positive control group. These data suggest that the activation of the apoptosis-related protein cleaved caspase- 3 is another process that contributes to the mechanisms by which MenSC-Ex inhibit liver apoptosis in D-GalN/LPS-induced FHF.

NK cells are innate immune cells. A variety of artificially synthesized and natural products have been tested 
to evaluate their ability to recruit and activate NK cells [51]. Microbial (especially viral) invasion, tumor metastasis, and environmental materials are also capable of recruiting and activating NK cells in particular tissues or organs $[52,53]$. Approximately $30-40 \%$ and $10-20 \%$ of the total number of MNC cells are NK cells in the livers of humans and mice, respectively $[54,55]$. Some recent reports have shown that NK cells are involved in the pathogenesis of human hepatitis and animal models of liver injury [56-59]. D-GalN/LPS-induced FHF is a macrophage-mediated model of septic hepatitis $[60,61]$. In recent years, a considerable amount of data has accumulated regarding the involvement of NK cells in the pathogenesis of sepsis, a systemic inflammatory process that is induced by bacterial lipopolysaccharide (LPS) [62]. According to data obtained by our group, D-GalN/LPS acts directly on NK cells to accelerate the increase in the number of NK cells in the injured liver. When cells or animals were treated with MenSC-Ex, the number of NK cells decreased. The reason that MenSC-Ex inhibited the increase in NK cells that was observed in the D-GalN/ LPS-only treated livers remains unknown.

Caspase-3 is the most important apoptosis-associated protein [63]. The activation of caspase-3 induces an enzymatic cascade in mammalian cells that is viewed as an 'executioner' of apoptosis [64]. In addition, the first step in hepatocellular apoptosis requires the activation of caspase-3 [65]. In the present study, mice treated with MenSC-Ex exhibited lower levels of caspase-3 protein in the liver than were observed in the D-GalN/LPS-treated group. This finding suggests that the caspase-3-mediated death receptor pathway is one of the main mechanisms by which D-GalN/LPS induce hepatocyte apoptosis. Studies aimed at exploring the role of apoptosis proteins in D-GalN/LPS-induced liver apoptosis may provide insights into potential mechanisms through which MenSCEx can be used to treat D-GalN/LPS-induced FHF.

Silymarin has the therapeutic effect of restoring the normal liver functions [66]. In our study, silymarin had the ability of improving liver function in FHF mice, and its regenerative capabilities were the same as MenSC-Ex.

Although we demonstrated that MenSC-Ex ameliorated D-GalN/LPS-induced FHF in mice and explored mechanisms that may potentially underlie this effect in the present study, there are still many challenges to overcome before applications involving MenSC-Ex can be used in clinical medical practice. For example, FHF occurs rapidly, and when patients present at the hospital, they are almost always in the terminal stage of the disease. FHF causes the massive death of liver cells, and humans are much larger than mice and most other model mammals. Therefore, when MenSC-Ex are used to treat this disease, appropriate attention must be paid to the dose. In this study, MenSC-Ex reduced apoptosis in hepatocellular cells and enhanced longevity in FHF mice. However, treatments that do not renew hepatocellular tissues may be insufficient for treating FHF. Therefore, identifying methods to use MenSC-Ex in therapeutics remains a necessity for developing these cells into clinically applicable strategies.

\section{Conclusions}

In this study, we present preliminary evidence demonstrating the potential of MenSC-Ex for reducing fulminant hepatic failure (FHF). These data suggest that MenSC-Ex transplantation deserves further study as an adjunctive or alternative approach for treating FHF.

\section{Additional files}

Additional file 1: List of the 120 cytokines (including the G6 human cytokine antibody array and the G7 human cytokine antibody array) that were evaluated using a Human Cytokine G1000 array. POS positive, NEG negative. (TIF $4786 \mathrm{~kb}$ )

\section{Abbreviations}

ALT: alanine aminotransferase; ANOVA: analysis of variance; AST: aspartate aminotransferase; BSA: bovine serum albumin; CCK-8: Cell Counting Kit-8; CFU-F: colony-forming unit-fibroblast; D-GalN: D-galactosamine; DLS: dynamic light scatter; ECL: enhanced chemiluminescence;

ELISA: enzyme-linked immunosorbent assays; FACS: fluorescence-activated cell sorting; FBS: fetal bovine serum; FHF: fulminant hepatic failure; H\&E: hematoxylin and eosin; HPLC: high-performance liquid chromatography; ICAM-1: intercellular cell adhesion molecule-1; IGFBP-6: insulin-like growth factorbinding protein 6; IL-6: interleukin 6; IL-8: interleukin 8; ITS: insulin, transferrin, selenium; LPS: lipopolysaccharide; MBPCs: menstrual blood progenitor cells; MenSC-Ex: human menstrual blood-derived stem cell-derived exosomes; MenSCs: human menstrual blood-derived stem cells; MNCs: liver mononuclear cells; MSC: mesenchymal stem cells; MWCO: molecular weight cutoff; NK: natural killer cells; PBS: phosphate-buffered saline; PMSF: phenylmethylsulfonyl fluoride; RT-PCR: reverse transcription-polymerase chain reaction; TEM: transmission electron microscopy; TNF-a: tumor necrosis factor-a; TUNEL: terminal deoxynucleotidyl transferase dUTP nick-end labeling

\section{Acknowledgements}

We thank Bo Chen, Lijun Chen, Yongjia Zhao, Yang Guo, and Yanling Wang for their suggestions and technical contributions to this study.

\section{Funding}

This work was funded by National High-Tech R\&D Program 863 Grant 2015AA020306.

\section{Availability of data and materials}

Additional file 1 List of the 120 cytokines (including the G6 human cytokine antibody array and the $\mathrm{G} 7$ human cytokine antibody array) that were evaluated using a Human Cytokine G1000 array. POS positive, NEG negative.

\section{Authors' contributions}

$L C$ and $C X$ designed this study. $L C$ performed the experiments, collected and analyzed the data, and wrote the manuscript. BX and XW contributed with critical review of data analyses. All authors have read and approved the final manuscript.

\section{Competing interests}

The authors declare that they have no competing interests.

Consent for publication

All authors provide consent for publication of this manuscript. 


\section{Ethics approval}

This study was submitted to and approved by The Tab of Animal Experimental Ethical Inspection of the First Affiliated Hospital, College of Medicine, Zhejiang University, approval number 2016-(288).

\section{Received: 13 August 2016 Revised: 28 November 2016}

Accepted: 7 December 2016 Published online: 23 January 2017

\section{References}

1. Van Thiel DH, Brems J, Nadir A, Idilman R, Colantoni A, Holt D, et al. Liver transplantation for fulminant hepatic failure. J Gastroenterol. 2002;37 Suppl 13:78-81.

2. Hishinuma I, Nagakawa J, Hirota K, Miyamoto K, Tsukidate K, Yamanaka T, et al. Involvement of tumor necrosis factor-alpha in development of hepatic injury in galactosamine-sensitized mice. Hepatology. 1990;12:1187-91.

3. Liaskou E, Wilson DV, Oo YH. Innate immune cells in liver inflammation. Mediators Inflamm. 2012;2012:949157.

4. Meng X, Ichim TE, Zhong J, Rogers A, Yin Z, Jackson J, et al. Endometrial regenerative cells: a novel stem cell population. J Transl Med. 2007;5:57.

5. Allickson J, Xiang C. Human adult stem cells from menstrual blood and endometrial tissue. J Zhejiang Univ Sci B. 2012;13:419-20.

6. Sugawara K, Hamatani T, Yamada M, Ogawa S, Kamijo S, Kuji N, et al. Derivation of human decidua-like cells from amnion and menstrual blood. Sci Rep. 2014;4:4599.

7. Alcayaga-Miranda F, Cuenca J, Luz-Crawford P, Aguila-Diaz C, Fernandez A Figueroa FE, et al. Characterization of menstrual stem cells: angiogenic effect, migration and hematopoietic stem cell support in comparison with bone marrow mesenchymal stem cells. Stem Cell Res Ther. 2015;6:32.

8. Gargett CE, Masuda H. Adult stem cells in the endometrium. Mol Hum Reprod. 2010;16:818-34.

9. Khoury M, Alcayaga-Miranda F, Illanes SE, Figueroa FE. The promising potential of menstrual stem cells for antenatal diagnosis and cell therapy. Front Immunol. 2014:5:205

10. Nikoo S, Ebtekar M, Jeddi-Tehrani M, Shervin A, Bozorgmehr M, Vafaei S, et al. Menstrual blood-derived stromal stem cells from women with and without endometriosis reveal different phenotypic and functional characteristics. Mol Hum Reprod. 2014;20:905-18.

11. Lin J, Xiang D, Zhang JL, Allickson J, Xiang C. Plasticity of human menstrual blood stem cells derived from the endometrium. J Zhejiang Univ Sci B. 2011;12:372-80.

12. Wu X, Luo Y, Chen J, Pan R, Xiang B, Du X, et al. Transplantation of human menstrual blood progenitor cells improves hyperglycemia by promoting endogenous progenitor differentiation in type 1 diabetic mice. Stem Cells Dev. 2014;23:1245-57.

13. Zhong Z, Patel AN, Ichim TE, Riordan NH, Wang H, Min WP, et al. Feasibility investigation of allogeneic endometrial regenerative cells. J Transl Med. 2009;7:15

14. Borlongan CV, Kaneko Y, Maki M, Yu SJ, Ali M, Allickson JG, et al. Menstrual blood cells display stem cell-like phenotypic markers and exert neuroprotection following transplantation in experimental stroke. Stem Cells Dev. 2010;19:439-52

15. Liu T, Huang Y, Zhang J, Qin W, Chi H, Chen J, et al. Transplantation of human menstrual blood stem cells to treat premature ovarian failure in mouse model. Stem Cells Dev. 2014;23:1548-57.

16. Rodrigues MC, Voltarelli J, Sanberg PR, Allickson JG, Kuzmin-Nichols N, Garbuzova-Davis S, et al. Recent progress in cell therapy for basal ganglia disorders with emphasis on menstrual blood transplantation in stroke. Neurosci Biobehav Rev. 2012;36:177-90.

17. Zhang Z, Wang JA, Xu Y, Jiang Z, Wu R, Wang L, et al. Menstrual blood derived mesenchymal cells ameliorate cardiac fibrosis via inhibition of endothelial to mesenchymal transition in myocardial infarction. Int J Cardiol. 2013;168:1711-4

18. Santamaria X, Massasa EE, Feng Y, Wolff E, Taylor HS. Derivation of insulin producing cells from human endometrial stromal stem cells and use in the treatment of murine diabetes. Mol Ther. 2011;19:2065-71.

19. Cui CH, Uyama T, Miyado K, Terai M, Kyo S, Kiyono T, et al. Menstrual bloodderived cells confer human dystrophin expression in the murine model of Duchenne muscular dystrophy via cell fusion and myogenic transdifferentiation. Mol Biol Cell. 2007;18:1586-94.

20. Thery C, Ostrowski M, Segura E. Membrane vesicles as conveyors of immune responses. Nat Rev Immunol. 2009;9:581-93.
21. Chen TS, Arslan F, Yin Y, Tan SS, Lai RC, Choo AB, et al. Enabling a robust scalable manufacturing process for therapeutic exosomes through oncogenic immortalization of human ESC-derived MSCs. J Transl Med. 2011:9:47.

22. Lai RC, Arslan F, Tan SS, Tan B, Choo A, Lee MM, et al. Derivation and characterization of human fetal MSCs: an alternative cell source for largescale production of cardioprotective microparticles. J Mol Cell Cardiol. 2010;48:1215-24.

23. Lai RC, Arslan F, Lee MM, Sze NS, Choo A, Chen TS, et al. Exosome secreted by MSC reduces myocardial ischemia/reperfusion injury. Stem Cell Res. 2010;4:214-22.

24. Feher J, Lengyel G. Silymarin in the prevention and treatment of liver diseases and primary liver cancer. Curr Pharm Biotechnol. 2012;13(1):210-7.

25. Shaker E, Mahmoud H, Mnaa S. Silymarin, the antioxidant component and Silybum marianum extracts prevent liver damage. Food Chem Toxicol. 2010:48(3):803-6.

26. Yamaza T, Miura Y, Bi Y, Liu Y, Akiyama K, Sonoyama W, Patel V, Gutkind S, Young M, Gronthos S, et al. Pharmacologic stem cell based intervention as a new approach to osteoporosis treatment in rodents. PLoS One. 2008;3(7):e2615.

27. Wang J, Sun R, Wei H, Dong Z, Gao B, Tian Z. Poly I:C prevents T cell-mediated hepatitis via an NK-dependent mechanism. J Hepatol. 2006:44:446-54.

28. Chen L, Su J, Yang C, Peng L, Wan Q, Wang L. Functional characterizations of RIG-I to GCRV and viral/bacterial PAMPs in grass carp Ctenopharyngodon idella. PLoS One. 2012;7:e42182.

29. Li T, Yan Y, Wang B, Qian H, Zhang $X$, Shen $L$, et al. Exosomes derived from human umbilical cord mesenchymal stem cells alleviate liver fibrosis. Stem Cells Dev. 2013;22:845-54.

30. Lin R, Wang S, Zhao RC. Exosomes from human adipose-derived mesenchymal stem cells promote migration through Wnt signaling pathway in a breast cancer cell model. Mol Cell Biochem. 2013;383:13-20.

31. Mou XZ, Lin J, Chen JY, Li YF, Wu XX, Xiang BY, et al. Menstrual bloodderived mesenchymal stem cells differentiate into functional hepatocyte-like cells. J Zhejiang Univ Sci B. 2013;14:961-72.

32. Togel F, Weiss K, Yang Y, Hu Z, Zhang P, Westenfelder C. Vasculotropic paracrine actions of infused mesenchymal stem cells are important to the recovery from acute kidney injury. Am J Physiol Renal Physiol. 2007:292:F1626-35.

33. Camussi G, Deregibus MC, Tetta C. Paracrine/endocrine mechanism of stem cells on kidney repair: role of microvesicle-mediated transfer of genetic information. Curr Opin Nephrol Hypertens. 2010;19:7-12.

34. Qin ZH, Xu JF, Qu JM, Zhang J, Sai $Y$, Chen CM, et al. Intrapleural delivery of MSCs attenuates acute lung injury by paracrine/endocrine mechanism. J Cell Mol Med. 2012;16:2745-53.

35. Meirelles Lda S, Fontes AM, Covas DT, Caplan Al. Mechanisms involved in the therapeutic properties of mesenchymal stem cells. Cytokine Growth Factor Rev. 2009:20:419-27.

36. Bruno S, Grange C, Deregibus MC, Calogero RA, Saviozzi S, Collino F, et al. Mesenchymal stem cell-derived microvesicles protect against acute tubular injury. J Am Soc Nephrol. 2009;20:1053-67.

37. Gatti S, Bruno S, Deregibus MC, Sordi A, Cantaluppi V, Tetta C, et al. Microvesicles derived from human adult mesenchymal stem cells protect against ischaemia-reperfusion-induced acute and chronic kidney injury. Nephrol Dial Transplant. 2011;26:1474-83.

38. Bruno S, Grange C, Collino F, Deregibus MC, Cantaluppi V, Biancone L, et al. Microvesicles derived from mesenchymal stem cells enhance survival in a lethal model of acute kidney injury. PLoS One. 2012;7:e33115.

39. Alison MR, Islam S, Lim S. Stem cells in liver regeneration, fibrosis and cancer: the good, the bad and the ugly. J Pathol. 2009;217(2):282-98.

40. Hong F, Kim WH, Tian Z, Jaruga B, Ishac E, Shen X, Gao B. Elevated interleukin-6 during ethanol consumption acts as a potential endogenous protective cytokine against ethanol-induced apoptosis in the liver: involvement of induction of BCl-2 and BCl-x(L) proteins. Oncogene. 2002;21(1):32-43.

41. Bosman MC, Reis CR, Schuringa JJ, Vellenga E, Quax WJ. Decreased affinity of recombinant human tumor necrosis factor-related apoptosis-inducing ligand (rhTRAIL) D269H/E195R to osteoprotegerin (OPG) overcomes TRAlL resistance mediated by the bone microenvironment. J Biol Chem. 2014;289(2):1071-8.

42. Boyce BF, Xing L. Functions of RANKL/RANK/OPG in bone modeling and remodeling. Arch Biochem Biophys. 2008;473(2):139-46.

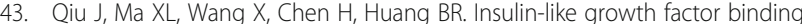
protein-6 interacts with the thyroid hormone receptor alpha1 and modulates the thyroid hormone-response in osteoblastic differentiation. Mol Cell Biochem. 2012;361(1-2):197-208. 
44. Micutkova L, Diener T, Li C, Rogowska-Wrzesinska A, Mueck C, Huetter E, Weinberger B, Grubeck-Loebenstein B, Roepstorff P, Zeng R, et al. Insulin-like growth factor binding protein-6 delays replicative senescence of human fibroblasts. Mech Ageing Dev. 2011;132(10):468-79.

45. Szekanecz Z, Shah MR, Harlow LA, Pearce WH, Koch AE. Interleukin-8 and tumor necrosis factor-alpha are involved in human aortic endothelial cell migration. The possible role of these cytokines in human aortic aneurysmal blood vessel growth. Pathobiology. 1994;62(3):134-9.

46. Van Damme J, Van Beeumen J, Opdenakker G, Billiau A. A novel, NH2terminal sequence-characterized human monokine possessing neutrophil chemotactic, skin-reactive, and granulocytosis-promoting activity. J Exp Med. 1988;167(4):1364-76.

47. Ren G, Zhao X, Zhang L, Zhang J, L'Huillier A, Ling W, Roberts Al, Le AD, Shi $S$, Shao $C$, et al. Inflammatory cytokine-induced intercellular adhesion molecule-1 and vascular cell adhesion molecule-1 in mesenchymal stem cells are critical for immunosuppression. J Immunol. 2010;184(5):2321-8.

48. D'Arcangelo D, Gaetano C, Capogrossi MC. Acidification prevents endothelial cell apoptosis by Axl activation. Circ Res. 2002;91(7):e4-12.

49. Cai J, Kehoe O, Smith GM, Hykin P, Boulton ME. The angiopoietin/Tie-2 system regulates pericyte survival and recruitment in diabetic retinopathy. Invest Ophthalmol Vis Sci. 2008:49(5):2163-71.

50. Li S, Hu GF. Emerging role of angiogenin in stress response and cell survival under adverse conditions. J Cell Physiol. 2012;227(7):2822-6.

51. Nakama T, Hirono S, Moriuchi A, Hasuike S, Nagata K, Hori T, et al. Etoposide prevents apoptosis in mouse liver with D-galactosamine/lipopolysaccharideinduced fulminant hepatic failure resulting in reduction of lethality. Hepatology. 2001;33:1441-50.

52. Wiltrout RH, Pilaro AM, Gruys ME, Talmadge JE, Longo DL, Ortaldo JR, et al. Augmentation of mouse liver-associated natural killer activity by biologic response modifiers occurs largely via rapid recruitment of large granular lymphocytes from the bone marrow. J Immunol. 1989;143:372-8.

53. Salazar-Mather TP, Orange JS, Biron CA. Early murine cytomegalovirus (MCMV) infection induces liver natural killer (NK) cell inflammation and protection through macrophage inflammatory protein 1alpha (MIP-1alpha)dependent pathways. J Exp Med. 1998;187:1-14.

54. Salazar-Mather TP, Lewis CA, Biron CA. Type I interferons regulate inflammatory cell trafficking and macrophage inflammatory protein 1alpha delivery to the liver. J Clin Invest. 2002;110:321-30.

55. Norris S, Collins C, Doherty DG, Smith F, McEntee G, Traynor O, et al. Resident human hepatic lymphocytes are phenotypically different from circulating lymphocytes. J Hepatol. 1998;28:84-90.

56. Goossens $\mathrm{PL}$, Jouin $\mathrm{H}$, Marchal G, Milon G. Isolation and flow cytometric analysis of the free lymphomyeloid cells present in murine liver. J Immunol Methods. 1990;132:137-44.

57. Dong Z, Wei H, Sun R, Hu Z, Gao B, Tian Z. Involvement of natural killer cells in Polyl:C-induced liver injury. J Hepatol. 2004;41:966-73.

58. Muhlen KA, Schumann J, Wittke F, Stenger S, Van Rooijen N, Van Kaer L, et al. NK cells, but not NKT cells, are involved in Pseudomonas aeruginosa exotoxin A-induced hepatotoxicity in mice. J Immunol. 2004;172:3034-41.

59. Dunn C, Brunetto M, Reynolds G, Christophides T, Kennedy PT, Lampertico P, et al. Cytokines induced during chronic hepatitis B virus infection promote a pathway for NK cell-mediated liver damage. J Exp Med. 2007;204:667-80.

60. Ahmad A, Alvarez F. Role of NK and NKT cells in the immunopathogenesis of HCV-induced hepatitis. J Leukoc Biol. 2004;76:743-59.

61. Wan JY, Gong X, Zhang L, Li HZ, Zhou YF, Zhou QX. Protective effect of baicalin against lipopolysaccharide/D-galactosamine-induced liver injury in mice by up-regulation of heme oxygenase-1. Eur J Pharmacol. 2008;587:302-8.

62. Jirillo E, Caccavo D, Magrone T, Piccigallo E, Amati L, Lembo A, et al. The role of the liver in the response to LPS: experimental and clinical findings. J Endotoxin Res. 2002;8:319-27.

63. Enari M, Sakahira H, Yokoyama H, Okawa K, Iwamatsu A, Nagata S. A caspase-activated DNase that degrades DNA during apoptosis, and its inhibitor ICAD. Nature. 1998;391:43-50.

64. Thornberny NA, Lazebnik Y. Caspases: enemies within. Science. 1998;281:1312-6.

65. Woo M, Hakem A, Elia AJ, Hakem R, Duncan GS, Patterson BJ, et al. In vivo evidence that caspase-3 is required for Fas-mediated apoptosis of hepatocytes. J Immunol. 1999;163:4909-16.

66. Pradhan SC, Girish C. Hepatoprotective herbal drug, silymarin from experimental pharmacology to clinical medicine. Indian J Med Res. 2006;124(5):491-504.

\section{Submit your next manuscript to BioMed Central and we will help you at every step:}

- We accept pre-submission inquiries

- Our selector tool helps you to find the most relevant journal

- We provide round the clock customer support

- Convenient online submission

- Thorough peer review

- Inclusion in PubMed and all major indexing services

- Maximum visibility for your research

Submit your manuscript at www.biomedcentral.com/submit 